\title{
Hypergraph list coloring and Euclidean Ramsey Theory
}

\author{
Noga Alon* $\quad$ Alexandr Kostochka ${ }^{\dagger}$
}

November 22, 2010

\begin{abstract}
A hypergraph is simple if it has no two edges sharing more than a single vertex. It is $s$-list colorable (or $s$-choosable) if for any assignment of a list of $s$ colors to each of its vertices, there is a vertex coloring assigning to each vertex a color from its list, so that no edge is monochromatic. We prove that for every positive integer $r$, there is a function $d_{r}(s)$ such that no $r$-uniform simple hypergraph with average degree at least $d_{r}(s)$ is $s$-list-colorable. This extends a similar result for graphs, due to the first author, but does not give as good estimates of $d_{r}(s)$ as are known for $d_{2}(s)$, since our proof only shows that for each fixed $r \geq 2, d_{r}(s) \leq 2^{c_{r} s^{r-1}}$. We use the result to prove that for any finite set of points $X$ in the plane, and for any finite integer $s$, one can assign a list of $s$ distinct colors to each point of the plane, so that any coloring of the plane that colors each point by a color from its list contains a monochromatic isometric copy of $X$.
\end{abstract}

AMS Subject Classification: 05C15, 05C35, 05C65.

Keywords: Hypergraphs, list coloring, average degree, Euclidean Ramsey Theory.

\section{Introduction}

\subsection{Background}

The list chromatic number (or choice number) $\chi_{\ell}(G)$ of a graph $G=(V, E)$ is the minimum integer $s$ such that for every assignment of a list $L_{v}$ of $s$ colors to each vertex $v$ of $G$, there is a proper vertex coloring of $G$ in which the color of each vertex is in its list. This notion was introduced independently by Vizing in [22] and by Erdős, Rubin and Taylor in [10]. In both papers the authors realized that this is a variant of usual coloring that exhibits several new properties, and that in general $\chi_{\ell}(G)$, which is always at least as large as the chromatic number of $G$, may be arbitrarily large even for graphs $G$ of chromatic number 2 .

\footnotetext{
${ }^{*}$ Sackler School of Mathematics and Blavatnik School of Computer Science, Tel Aviv University, Tel Aviv 69978, Israel and Institute for Advanced Study, Princeton, New Jersey, 08540, USA. E-mail address: nogaa@tau.ac.il. Research supported in part by an ERC Advanced grant, by a USA-Israeli BSF grant, by the Oswald Veblen Fund and by the Bell Companies Fellowship.

${ }^{\dagger}$ Department of Mathematics, University of Illinois, Urbana, IL, 61801, USA and Institute of Mathematics, Novosibirsk, Russia. Email: kostochk@math.uiuc.edu. Research supported in part by NSF grants DMS-0650784 and DMS-0965587 and by grant 08-01-00673 of the Russian Foundation for Basic Research.
} 
It is natural to extend the notion of list coloring to hypergraphs, and indeed this has been done, among other places, in [21]. The list chromatic number $\chi_{\ell}(H)$ of a hypergraph $H$ is the minimum integer $s$ such that for every assignment of a list of $s$ colors to each vertex of $H$, there is a vertex coloring of $H$ assigning to each vertex a color from its list, with no monochromatic edges.

An intriguing property of list coloring of graphs, which is not shared by ordinary vertex coloring, is the result proved by the first author in $[2,3]$ that the list chromatic number of any (simple) graph with a large average degree is large. Indeed, it is shown in [3] that the list chromatic number of any graph with average degree $d$ is at least $\left(\frac{1}{2}-o(1)\right) \log _{2} d$, where the $o(1)$-term tends to zero as $d$ tends to infinity. Ramamurthi [20] asked whether a similar statement holds for $r$-uniform hypergraphs ( $r$-graphs, for short). For $r \geq 3$, there is no nontrivial lower bound on the list chromatic number of an $r$-graph in terms of its average degree. To see this, consider, for example, a perfect matching $M$ consisting of $n / 2$ isolated (graph) edges on a set of $n$ vertices, and let $H=H(M)$ be the $r$-graph on this set of vertices consisting of all $r$-edges containing at least one edge of $M$. Then the degree of every vertex of $H$ is greater than $\left(\begin{array}{l}n-2 \\ r-2\end{array}\right)$, and yet its list chromatic number is 2. More generally, one can replace the matching $M$ by any graph $G$ of list chromatic number bounded by $t$, and consider the hypergraph $H$ of all $r$-edges containing at least one edge of $G$, which clearly satisfies $\chi_{\ell}(H) \leq t$. For example, if $n$ is divisible by $2 g$ and we replace $M$ with the graph $G$ that is the disjoint union of $n / 2 g$ complete bipartite graphs $K_{g, g}$, then the degree of every vertex of the corresponding $r$-graph

$H=H(G)$ is at least $(1+o(1)) g\left(\begin{array}{c}n \\ r-2\end{array}\right)$, where for any fixed $r$ and $g$, the $o(1)$-term tends to zero as $n$ tends to infinity, whereas the list chromatic number of $H$ is only $(1+o(1)) \log _{2} g$.

\subsection{Simple hypergraphs}

The dense hypergraphs of bounded list chromatic number in the above examples are not simple. Recall that a hypergraph is called simple if every two of its distinct edges share at most one vertex. In the present paper we prove that the result of [3] can be extended to simple $r$-graphs. This is stated in the following theorem.

Theorem 1.1 For every fixed $r \geq 2$ and $s \geq 6 r$, there is $d=d(r, s)$, such that the list chromatic number of any simple $r$-graph with $n$ vertices and nd edges is greater than $s$.

This extends the main result of [3] (which is the case $r=2$ of the above theorem), as well as that of Haxell and Pei, who proved in [12] that the list chromatic number of any Steiner Triple System on $n$ vertices is at least $s$, for all $n \geq n_{0}(s)$. After obtaining our results we learned that Haxell and Verstraete [13] proved a similar result for the special case of $d$-regular 3 -uniform simple hypergraphs.

It is worth noting that the theorem provides a linear time algorithm for computing, for a given input simple $r$-graph, a number $s$ such that its list chromatic number is at least $s$ and at most $f(s)$ for some explicit function $f$. There is no such known result for ordinary coloring, and it is known that there cannot be one under some plausible hardness assumptions in Complexity Theory - see [6] and a few additional related comments in the last section of the present paper. 
In order to prove Theorem 1.1, we will prove a stronger statement - if the average degree of an $r$-graph is sufficiently large, then there is a way to assign lists of $s$ colors, from a set of not too many colors, to all the vertices so that in any vertex coloring from the lists there are many monochromatic edges. This statement seems to be crucial for the proof, and is stated in the following theorem.

Theorem 1.2 For every fixed $r \geq 2$, there are functions $d_{r}(s), R_{r}(s) \geq s$ and $0<\delta_{r}(s) \leq 1 / R_{r}(s)$ such that the following holds. For each $s \geq 6 r$, every $d>d_{r}(s)$ and every $n$-vertex simple $r$-graph $G$ with $d n$ edges, there is an assignment $L$ of lists of size $s$ from the set $\left[R_{r}(s)\right]=\left\{1,2, \ldots, R_{r}(s)\right\}$ to the vertices of $G$ such that for every coloring of the vertices of $G$ from these lists, the number of monochromatic edges in $G$ is at least $\delta_{r}(s) \cdot d n$.

\subsection{A geometric application}

A well known problem of Hadwiger and Nelson is that of determining the minimum number of colors required to color the points of the Euclidean plane so that no two points at distance 1 have the same color. Hadwiger showed already in 1945 that 7 colors suffice, and L. Moser and W. Moser noted [19] that 3 colors do not suffice. These bounds have not been improved, despite a considerable amount of effort by various researchers, see [15, pp. 150-152] and the references therein for more on the history of the problem.

A more general problem is considered in [7], [8], [9], where the main question is the investigation of finite point sets $K$ in the Euclidean space for which any coloring of an Euclidean space of a sufficiently high dimension $d \geq d_{0}(K, r)$ by $r$ colors must contain a monochromatic copy of $K$. The main conjecture is that this holds for any set $K$ that can be embedded in a sphere.

The situation is different for list coloring. As described in [16], for every integer $s$ there is an assignment of a list of $s$ colors to each point of the plane such that in any coloring of the plane that colors each point by a color from its list there are two points of distance 1 having the same color. This can be deduced from the main result of [3]. As a corollary of our results here, we prove the following.

Theorem 1.3 For any finite set $X$ in the Euclidean plane and for any positive integer $s$, there is an assignment of a list of size $s$ to every point of the plane, such that whenever we color the points of the plane from their lists, there is a monochromatic isometric copy of $X$.

\subsection{Organization}

In the next section we outline the proof of the main result. One of the difficulties in this proof is the problem of handling $r$-graphs in which the minimum degree is much smaller than the average degree. To overcome this obstacle, we prove in Section 3 a simple decomposition result showing that any $r$-graph $H$ contains a subgraph $H^{\prime}$ with at least, say, half the edges of $H$, that can be decomposed into large $r$-graphs each having average degree at most $r$ times the minimum degree. The proof of Theorem 1.2 for $r=2$ is described in Section 4 and the proof for general $r$ in Section 5 . 
Section 6 is devoted to the geometric application, and the final section contains some concluding remarks and open problems.

\section{An outline of the proof}

The proof of Theorem 1.2 applies induction on $r$. Although it is possible to start the induction with the trivial case $r=1$, we prefer to start with $r=2$, as this supplies a better estimate for the general case. In this section we sketch the proof for the base case $r=2$ and for the case $r=3$. The proof of the induction step for general $r$ is similar to the proof for $r=3$, with a few additional technical complications described in Section 5.

First, consider the case $r=2$. Let $G=(V, E)$ be a graph with $n$ vertices and $n d$ edges. We (try to) start as in [3]: choose a random set $B$ of about $n / \sqrt{d}$ vertices and assign a random list of size $s$ out of a set $S$ of $2 s-1$ colors to each vertex of $B$. Say that a vertex $v \in V \backslash B$ is normal, if every subset of size $s$ of $S$ is assigned to at least one of $B$-neighbors of $v$. A simple computation shows that with positive (and in fact high) probability there are many normal vertices in $V \backslash B$. Fix such a choice of the set $B$ and the lists for its vertices. Note now that for each fixed choice of a coloring $f$ of the vertices of $B$ from their lists, at least $s$ distinct colors appear on the $B$-neighbors of any normal vertex $v$. If we now assign a random list to a normal vertex $v$, then with probability at least $\left(\begin{array}{c}2 s-1 \\ s\end{array}\right)^{-1}>4^{-s}$ it will be a forbidden list, that is, it will consist only of colors assigned by $f$ to its neighbors, showing that the coloring $f$ of the vertices in $B$ cannot be extended to a proper list coloring of the whole graph. There are only $s^{|B|}$ possible colorings of the vertices of $B$ from their lists, and the probability that no vertex $v$ gets a forbidden list is small enough to ensure that this will not happen for any of these colorings. This argument suffices to show that the list chromatic number of $G$ exceeds $s$. However, our objective is to prove a stronger result (needed for proceeding with the case of 3-uniform hypergraphs): there is an assignment of lists of size $s$ to the vertices of $G$ such that in any coloring from these lists, at least a $\delta_{2}(s)$-fraction of the edges is monochromatic. With some care, the proof described above does imply that in any such coloring there are at least some $\delta_{2}(s)$-fraction of the vertices such that for each such vertex $v$, at least a $\delta_{2}(s)$-fraction of the edges incident with $v$ are monochromatic. However, since the minimum degree in $G$ may be much smaller than its average degree, this does not suffice.

In order to deal with this case, we first show that $G$ contains a subgraph consisting of at least half the edges of $G$ that can be decomposed into a collection of pairwise edge-disjoint graphs $G_{i}$, such that each $G_{i}$ is large and its minimum degree (which is also large) is at least half of its average degree. It turns out that we can now select a set of vertices $B$ and an assignment of lists to its members so that $B$ and its lists will be good enough to handle simultaneously all graphs $G_{i}$. This works because the probabilistic estimates are strong enough to ensure that the events corresponding to all graphs $G_{i}$ hold. Thus each $G_{i}$ will contribute its share of monochromatic edges, implying the desired result for $r=2$.

Given the result for $r=2$, we sketch the proof of the case $r=3$, that is, the case of simple 3 -graphs. The decomposition result, that holds for 3-graphs as well, enables us to focus on one 
member of the decomposition, which is a simple 3-graph in which the minimum degree is not much smaller than the average degree. Given such a simple 3 -graph $G$ with $n$ vertices and $n d$ edges satisfying this condition, we select in it a large number $M$ of random pairwise disjoint sets $V_{1}, V_{2}, \ldots, V_{M}$ of vertices, each of size roughly $\frac{n}{d^{1 / 4}}$. With high probability many of the vertices $v$ of $G$ not selected into any of these sets, have a (graph-)edge $e_{j, v}$ in each $V_{j}$, such that $e_{j, v} \cup v$ is an edge of $G$. The assumption that $G$ is simple implies that all these 2-edges $e_{j, v}$ are pairwise distinct, that is, $e_{j, v} \neq e_{j, w}$ if $v \neq w$.

We now apply the induction hypothesis and assign lists of $s$ colors to the vertices in $\cup_{j} V_{j}$ so that for each fixed $j$, in each coloring of these vertices from their lists, a $\delta_{2}(s)$-fraction of all edges $e_{j, v}$ is monochromatic. The sets of colors used for the different sets $V_{j}$ are pairwise disjoint, to ensure that a vertex $v$ for which $e_{j, v}$ is monochromatic for many different values of $j$ will have many distinct forbidden colors. A double counting ensures now that in any coloring of the vertices in $\cup_{j} V_{j}$ from their lists there is at least a $\delta_{3}^{\prime}(s)$-fraction of the vertices not in $\cup_{j} V_{j}$ for which there are at least $s$ forbidden colors. We can now assign random lists to all remaining vertices and proceed as in the case $r=2$ (here, too, the probabilistic estimates are strong enough to handle all hypergraphs in the decomposition simultaneously).

This completes the outline. The details are described in the following three sections.

\section{A decomposition result for $r$-graphs}

In this section we prove the following.

Lemma 3.1 Let $D$ and $d$ be positive integers satisfying $d \geq 4 D$. Let $G=(V, E)$ be an r-graph with $n$ vertices and $n d$ edges. Then there is a family of pairwise edge disjoint subgraphs $G_{i}=\left(U_{i}, E_{i}\right)$ of $G, 1 \leq i \leq q$, satisfying the following five conditions.

(i) $U_{q} \subseteq U_{q-1} \subseteq U_{q-2} \subseteq \ldots \subseteq U_{1}$,

(ii) $\left|U_{q}\right| \geq \sqrt[r]{n d / 4}$

(iii) The minimum degree of each of the $r$-graphs $G_{i}$ is at least $D$,

(iv) $\left|E_{i}\right| \leq\left|U_{i}\right| \cdot D$ for all $1 \leq i \leq q$, and

(v) $\left|\bigcup_{i=1}^{q} E_{i}\right| \geq \frac{1}{2}|E|$.

Proof: Starting with $G$, as long as there is a vertex of degree less than $D$ in $G$, omit it until we reach an $r$-graph with minimum degree at least $D$. Let $U_{1}$ be its vertex set. To construct $G_{1}$, for each vertex $u \in U_{1}$ pick an arbitrary set of $D$ edges of $G\left[U_{1}\right]$ incident with $u$, and let all these edges belong to $G_{1}$. By construction, $G_{1}$ has minimum degree at least $D$, and has at most $\left|U_{1}\right| D$ edges. We now remove all the edges of $G_{1}$ from $G$ and proceed in the same manner. Namely, as long as there is a vertex of degree less than $D$ in (the remaining part of) $G$, omit it until we reach an $r$-graph with minimum degree at least $D$. Let $U_{2}$ be its vertex set, and note that $U_{2} \subseteq U_{1}$. For each vertex $u \in U_{2}$ pick an arbitrary set of $D$ edges incident with $u$, and let all these edges belong to $G_{2}$. Again, $G_{2}$ has minimum degree at least $D$, and has at most $\left|U_{2}\right| D$ edges. Remove all the edges of $G_{2}$ and proceed to the next step. 
Continuing in this manner until the number of remaining vertices of $G$ becomes less than $\sqrt[r]{n d / 4}$, we obtain a sequence of $r$-graphs $G_{1}, G_{2}, \ldots, G_{q}$. The construction ensures that properties (i),(ii),(iii) and (iv) hold. In addition, the edges of $G$ that do not belong to any of the graphs $G_{i}$ are either edges incident to one of the vertices deleted during the process when its degree was less than $D$ (there are fewer than $n D$ such edges), or edges contained in the set of remaining vertices when the number of such vertices became smaller than $\sqrt[r]{n d / 4}$ (there are fewer than $n d / r ! 4 \leq n d / 8$ such edges). Since by definition $d>4 D$, property (v) holds as well, showing that there are indeed $r$-graphs $G_{i}$ as claimed.

Observe that by Lemma 3.1(iv) and (v),

$$
D \sum_{i=1}^{q}\left|U_{i}\right| \geq \sum_{i=1}^{q}\left|E_{i}\right| \geq \frac{1}{2}|E|=\frac{n d}{2}
$$

So, denoting $n_{i}:=\left|V_{i}\right|$, we have

$$
\sum_{i=1}^{q} n_{i} \geq \frac{n d}{2 D}
$$

\section{List coloring of graphs}

In this section, we apply Lemma 3.1 for ordinary graphs (2-graphs). We prove the basic case $r=2$ of Theorem 1.2 in the following form.

Theorem 4.1 There exists a positive function $\delta(s)>2^{-6 s-11} / s^{2}$ such that for every $s \geq 12$ and every $d>4 \cdot\left(s 4^{s+2}\right)^{2}$, for any graph $G=(V, E)$ with $n$ vertices and $n d$ edges, there is an assignment of a list $L_{v}$ of size $s$ from a set of $2 s-1$ colors to each vertex $v \in V$ such that for every coloring assigning to each vertex a color from its list, the number of monochromatic edges is at least $\delta(s) \cdot d n$.

Proof: Let $G=(V, E)$ be a graph with $n$ vertices and $n d$ edges. Apply Lemma 3.1 with $D=\left(s 4^{s+2}\right)^{2}$ to $G$. Let $G_{1}, \ldots, G_{q}$ be the edge-disjoint subgraphs of $G$ guaranteed by the lemma. For each $i, 1 \leq i \leq q$, define $n_{i}=\left|U_{i}\right|$.

For a subset $B$ of vertices of $G$ and an assignment of a list $L_{v}$ of $s$ colors from the set $S=$ $\{1,2, \ldots, 2 s-1\}$ to each vertex $v$ of $B$, and for an integer $i, 1 \leq i \leq q$, we say that a vertex $u$ of $G$ is $i$-normal if it belongs to $U_{i} \backslash B$, the number of its neighbors in $G_{i}$ that lie in $U_{i} \cap B$ is at least $0.5 \sqrt{D}$, and for every subset $L \subseteq S$ of size $|L|=s$, there is at least one neighbor $v$ of $u$ in $G_{i}$ that lies in $B$ and whose list $L_{v}$ is $L$. Let $T_{i} \subseteq U_{i}$ be the set of all $i$-normal vertices.

First, we claim that there is a subset $B$ of vertices of $G$ and an assignment of lists to its vertices such that

(a) For each $i,\left|B \cap U_{i}\right| \leq \frac{2 n_{i}}{\sqrt{D}}$, and

(b) $\sum_{i=1}^{q}\left|T_{i}\right|>0.9 \sum_{i=1}^{q} n_{i}$.

Indeed, let $B$ be a random set chosen by picking each vertex of $G$ randomly and independently with probability $1 / \sqrt{D}$. By the Chernoff bound (see [1, Appendix A1] or [14, p.26, (2.5)]) the 
probability that $\left|B \cap U_{i}\right|>\frac{2 n_{i}}{\sqrt{D}}$ is at most $\exp \left\{-3 n_{i} / 8 \sqrt{D}\right\}$. Since $n_{i}>\sqrt{n d} / 2 \geq \sqrt{n D}$ for all $i$, this probability is at most $\exp \{-3 \sqrt{n} / 8\}$, and each $G_{i}$ has at least $D n_{i} / 2 \geq D \sqrt{n}$ edges. So,

$$
q \leq d n / D \sqrt{n}<n^{3 / 2} / D
$$

and hence the probability that (a) does not hold is at most

$$
\exp \{-3 \sqrt{n} / 8\} q \leq \exp \{-3 \sqrt{n} / 8\} n^{3 / 2} / D .
$$

By Lemma 3.1(iii), for every fixed vertex $u \in U_{i}$, the probability that at least $\sqrt{D} / 2$ vertices in $B$ are neighbors of $u$ in $G_{i}$ is at least

$$
1-\exp \{-\sqrt{D} / 8\}=1-\exp \left\{-2 s 4^{s}\right\} \geq 1-\exp \left\{-4^{s}\right\} .
$$

For $s \geq 12$ this is greater than 0.999 . Let $T_{i}^{\prime}$ be the set of vertices of $G_{i}$ that do not lie in $B$ and have, in $G_{i}$, at least $\sqrt{D} / 2$ neighbors in $B$. By linearity of expectation and by Markov's Inequality, with probability at least $1 / 2$ we have

$$
\sum_{i=1}^{q}\left(\left|T_{i}^{\prime}\right|+\left|B \cap U_{i}\right|\right) \geq 0.99 \sum_{i=1}^{q} n_{i}
$$

so with positive probability both (3) and (a) hold. Fix a set $B$ with these properties. By (3),

$$
\sum_{i=1}^{q}\left|T_{i}^{\prime}\right| \geq 0.99 \sum_{i=1}^{q} n_{i}-\sum_{i=1}^{q}\left|B \cap U_{i}\right| \geq \sum_{i=1}^{q} n_{i}\left(0.99-\frac{2}{\sqrt{D}}\right) \geq \sum_{i=1}^{q} n_{i}\left(0.99-\frac{1}{4^{s+2}}\right) \geq 0.97 \sum_{i=1}^{q} n_{i} .
$$

Assign to each vertex of $B$ a list which is a random subset of size $s$ of $S$. If a vertex $u$ belongs to $T_{i}^{\prime}$, then the probability that it is not $i$-normal is at most

$$
\left(\begin{array}{c}
2 s-1 \\
s
\end{array}\right)\left(1-\left(\begin{array}{c}
2 s-1 \\
s
\end{array}\right)^{-1}\right)^{\sqrt{D} / 2} \leq 4^{s}\left(1-4^{-s}\right)^{8 s 4^{s}} \leq 4^{s} e^{-8 s} .
$$

For $s \geq 12$ this is less than 0.01, implying the existence of the required lists for the vertices of $B$ by linearity of expectation (and the fact that $0.97 \cdot 0.99>0.9$ ).

Fix $B$ and lists $L_{v}$ for each $v \in B$ satisfying (a) and (b) above. Now we assign to all other vertices of $G$ lists that are random subsets of $S$ of size $s$. To complete the proof, we will show that with positive probability for every coloring of the vertices of $G$ from their lists, the number of monochromatic edges is at least $\delta(s) d n$.

For each $i$-normal vertex $v$, and for any assignment $c: B \cap U_{i} \mapsto S$ of colors to the vertices of $B \cap U_{i}$ from their lists, there are at least $s$ colors that appear on the neighbors of $v$ in $G_{i}$ that lie in $B$. Fix an assignment $f$ of colors to the vertices in $B \cap U_{i}$ from their lists. If $v$ is $i$-normal and if we now assign a random list $L \subseteq S$ of size $s$ to $v$, then the probability of the event $C(f, v, i)$ that $L$ contains only colors assigned to the $B$-neighbors of $v$ in $G_{i}$ is at least $\left(\begin{array}{c}2 s-1 \\ s\end{array}\right)^{-1}>4^{-s}$.

Let $I:=\left\{i \in[q]:\left|T_{i}\right|>0.5 n_{i}\right\}$. By (b) and the definition of $I$,

$$
\frac{9}{10} \sum_{i=1}^{q} n_{i}<\sum_{i=1}^{q}\left|T_{i}\right|=\sum_{i \in I}\left|T_{i}\right|+\sum_{i \in[q]-I}\left|T_{i}\right| \leq \sum_{i \in I}\left|T_{i}\right|+\frac{1}{2} \sum_{i \in[q]-I} n_{i} \leq \sum_{i \in I}\left|T_{i}\right|+\frac{1}{2} \sum_{i=1}^{q} n_{i}-\frac{1}{2} \sum_{i \in I} n_{i} .
$$


It follows that $0.4 \sum_{i=1}^{q} n_{i}<\sum_{i \in I}\left|T_{i}\right|-\frac{1}{2} \sum_{i \in I} n_{i} \leq \frac{1}{2} \sum_{i \in I}\left|T_{i}\right|$ and hence

$$
\sum_{i \in I}\left|T_{i}\right| \geq 0.8 \sum_{i=1}^{q} n_{i}
$$

If $i \in I$, the probability of the event $C^{\prime}(f, i)$ that $C(f, v, i)$ occurs for fewer than $0.5\left|T_{i}\right| 4^{-s}$ vertices $v \in T_{i}$ is very small. Indeed, by the Chernoff bound (see [1, Appendix A1, Theorem A.1.13] or [14, p.26, (2.6)]), it is at most $e^{-\left|T_{i}\right| 2^{-2 s-3}}$. So, if $i \in I$, then the probability that $C^{\prime}(f, i)$ occurs for at least one assignment $f$ of colors from the lists to the vertices in $B \cap U_{i}$ is at most

$$
e^{-\left|T_{i}\right| 2^{-2 s-3}} s^{\left|B \cap U_{i}\right|} \leq \exp \left\{\frac{2 n_{i}}{\sqrt{D}} \ln s-0.5 n_{i} 2^{-2 s-3}\right\}=\exp \left\{\frac{n_{i}}{4^{s+2}}\left(\frac{2 \ln s}{s}-1\right)\right\} .
$$

When $s \geq 12$, this is at most

$$
\exp \left\{-0.5 n_{i} 4^{-s-2}\right\} \leq \exp \left\{-0.5 \sqrt{n d / 4} \cdot 4^{-s-2}\right\}<\exp \left\{-0.5 \sqrt{n}\left(s 4^{s+2}\right) 4^{-s-2}\right\} \leq e^{-6 \sqrt{n}} .
$$

So, by (2), the probability that $C^{\prime}(f, i)$ occurs for at least one choice of $i$ and $f$ is at most

$$
q e^{-6 \sqrt{n}} \leq \frac{n^{3 / 2}}{D} e^{-6 \sqrt{n}} \leq \frac{n^{3 / 2}}{\left(s 4^{s+2}\right)^{2}} e^{-6 \sqrt{n}} \leq 1 / 2
$$

for every $n$. It follows that with positive probability none of $C^{\prime}(f, i)$ occurs at all. Fix a list assignment $L$ for $G$ such that none of $C^{\prime}(f, i)$ occurs for any coloring $f$ of $B$ from the list $L$. This means that for each extension $f^{\prime}$ to the whole $G$ of any such coloring $f$ and every $i \in I$, the number of vertices $v \in T_{i}$ contained in monochromatic edges in $E_{i}$ connecting $v$ with $B$ is at least $0.5\left|T_{i}\right| 4^{-s}$. Thus by (5) and (1) and the definition of $D$, the total number of monochromatic edges in any coloring of $V$ from $L$ is at least

$$
\sum_{i \in I} 0.5\left|T_{i}\right| 4^{-s} \geq 0.4 \cdot 4^{-s} \sum_{i=1}^{q} n_{i} \geq 0.4 \cdot 4^{-s} \frac{n d}{2 D}=0.2 \cdot 4^{-s} \frac{n d}{\left(s 4^{s+2}\right)^{2}}=0.2 \frac{n d}{s^{2} 2^{6 s+8}},
$$

which gives the desired result.

\section{Coloring simple hypergraphs}

In this section we prove Theorem 1.2 for all $r$. We apply induction on $r$. Theorem 4.1 yields the base case $r=2$ with $d_{2}(s)=4 \cdot\left(s 4^{s+2}\right)^{2}, R_{2}(s)=2 s-1$, and $\delta_{2}(s)=2^{-6 s-11} / s^{2}$. For $r \geq 3$, we will prove the theorem using

$$
\delta_{r}(s):=2^{-3^{r} r ! s^{r-1}}, \quad d_{r}(s):=\left(\delta_{r}(s)\right)^{-3}, \quad R_{r}(s):=\frac{4 s}{\delta_{r-1}^{2}(s)} .
$$

Let $r \geq 3, s \geq 6 r$ and $d>d_{r}(s)$. Let $G$ be a simple $r$-graph with $n$ vertices and $n d$ edges. 
Remark 1. Let $k * G$ be the $r$-graph consisting of $k$ vertex-disjoint copies of $G$. Then $k * G$ is a simple $r$-graph with the same average degree as $G$. Moreover, the statement of Theorem 1.2 holds for $k * G$ if and only if it holds for $G$. So, we may assume that $n$ is large, and in particular,

$$
\exp \{\sqrt[r]{n}\} \geq d n^{4}
$$

Put $M=\frac{4 s}{\delta_{r-1}(s)}, X=M^{2 s+2}$ and $D=X^{r-1}=M^{(2 s+2)(r-1)}$. By $(6)$,

$$
D X=X^{r}=M^{2 r(s+1)}=\left(\frac{4 s}{\delta_{r-1}(s)}\right)^{2 r(s+1)}=\left(2^{3^{r-1}(r-1) ! s^{r-2}+\log _{2} 4 s}\right)^{2 r(s+1)} .
$$

Since for $r \geq 3$ and $s \geq 6 r \geq 18,3^{r-1}(r-1) ! s^{r-2} \geq 10 \log _{2} 4 s$, we have

$$
D X \leq\left(2^{1.1 \cdot 3^{r-1}(r-1) ! s^{r-2}}\right)^{2 r(s+1)}=2^{1 \cdot 1 \cdot 3^{r-1} r ! s^{r-2} 2(s+1)}<2^{3^{r} r ! s^{r-1}}=\left(\delta_{r}(s)\right)^{-1}=\left(d_{r}(s)\right)^{1 / 3} .
$$

Also by (6) and the definition of $M$, we have

$$
M \geq R_{r-1}(s)
$$

Let $G_{1}=\left(U_{1}, E_{1}\right), \ldots, G_{q}=\left(U_{q}, E_{q}\right)$ be the edge-disjoint $r$-graphs guaranteed by Lemma 3.1. Let $n_{i}=\left|U_{i}\right|$ for $i=1, \ldots, q$.

Choose randomly disjoint subsets $V_{1}, V_{2}, \ldots, V_{M}$ of $V$, where each vertex, randomly and independently, is chosen to lie in $V_{j}$ with probability $\frac{2 \ln M}{D^{1 /(r-1)}}=\frac{2 \ln M}{X}$. For each vertex $v \in V$, the $i$-link of $v$, denoted $l_{i}(v)$, is the set of all $(r-1)$-edges of the form $e-\{v\}$ for all edges $e \in E_{i}$ that contain $v$. Note that for each $i$, if $v \in U_{i}$, then $l_{i}(v)$ is an $(r-1)$-matching of size at least $D$ (as $G$ is simple). Say that a vertex $v \in U_{i}$ is $i$-good if for every $j \in\{1, \ldots, M\}$ some edge of $l_{i}(v)$ is contained in $V_{j}$, and call an $i$-good vertex $i$-great if it does not lie in $\cup_{j=1}^{M} V_{j}$. By the Chernoff bound (see [1, Appendix A1] or [14, p.26, (2.5)]), the probability of the event $B(G)$ that the size of at least one $V_{j} \cap U_{i}$ exceeds $\frac{2 n_{i}(2 \ln M)}{X}$ is at most $M \sum_{i=1}^{q} \exp \left\{-3 n_{i} \ln M / 4 X\right\}$. Since $G$ is simple, $q<n^{2}$. So, by Lemma 3.1(ii) and (7),

$$
\mathbf{P}(B(G)) \leq q M \exp \left\{-3 \sqrt[r]{\frac{n d}{4}} \frac{\ln M}{4 X}\right\}<n^{2} M \exp \left\{-\sqrt[r]{n} X^{\frac{2 r-2}{r}} \frac{\ln M}{4 X}\right\}<n^{2} M^{1-\sqrt[r]{n} X^{1 / 3} / 4}<0.01 .
$$

The probability that a vertex $v \in U_{i}$ is not $i$-good is at most

$$
M\left(1-\left(\frac{2 \ln M}{X}\right)^{r-1}\right)^{D} \leq M \exp \left\{-(2 \ln M)^{2} \frac{D}{X^{r-1}}\right\}=M \exp \left\{-(2 \ln M)^{2}\right\}<0.001 .
$$

Hence with probability at least 0.9 there are at least $0.99 \sum_{i=1}^{q} n_{i}$ vertices that are $i$-good for some $i$ (counted with multiplicities). So by (10), with probability at least $1 / 2$ this holds and $B(G)$ does not hold. Fix such sets $V_{j}$. With this choice, if $T_{i}$ is the set of $i$-great vertices in $G_{i}$, then

$$
\sum_{i=1}^{q}\left|T_{i}\right| \geq 0.99 \sum_{i=1}^{q} n_{i}-\sum_{i=1}^{q} \sum_{j=1}^{M}\left|V_{j} \cap U_{i}\right| \geq \sum_{i=1}^{q} n_{i}\left(0.99-M \frac{4 \ln M}{X}\right)>0.9 \sum_{i=1}^{q} n_{i} .
$$


Let $I:=\left\{i \in[q]:\left|T_{i}\right|>0.5 n_{i}\right\}$. Similarly to deriving (5), (11) yields

$$
\sum_{i \in I}\left|T_{i}\right| \geq 0.8 \sum_{i=1}^{q} n_{i} .
$$

Now, for each pair $(v, i)$, where $i \in I$ and $v \in T_{i}$, keep in $G_{i}$ exactly $M$ edges containing $v$, one edge, call it $e(v, i, j)$, for each $j, 1 \leq j \leq M$, with $e(v, i, j)-\{v\} \subseteq V_{j} \cap U_{i}$, and omit all other $r$-edges from $G_{i}$. Let $G_{i}^{\prime}$ be the resulting hypergraph, and let $G^{\prime}=\bigcup_{i \in I} G_{i}^{\prime}$. For $j=1, \ldots, M$ and $i \in I$, let $G_{i}^{\prime \prime}(j)$ be the $(r-1)$-uniform hypergraph with $V\left(G_{i}^{\prime \prime}(j)\right)=V_{j} \cap U_{i}$ and $E\left(G_{i}^{\prime \prime}(j)\right):=$ $\left\{e \cap V_{j} \cap U_{i}: e \in E\left(G_{i}^{\prime}\right)\right.$ and $\left.\left|e \cap V_{j}\right|=r-1\right\}$. Let $G^{\prime \prime}(j)=\bigcup_{i \in I} G_{i}^{\prime \prime}(j)$ and $G_{i}^{\prime \prime}=\bigcup_{j=1}^{M} G_{i}^{\prime \prime}(j)$. Let $G^{\prime \prime}:=\bigcup_{j=1}^{M} G^{\prime \prime}(j)=\bigcup_{i \in I} G_{i}^{\prime \prime}$. Then $\left|E\left(G_{i}^{\prime \prime}(j)\right)\right|=\left|T_{i}\right|>0.5 n_{i}$ for each $i \in I$ and $j \in\{1, \ldots, M\}$, and by (12) and (1) for every $j=1, \ldots, M$,

$$
\left|E\left(G^{\prime \prime}(j)\right)\right|=\sum_{i \in I}\left|E\left(G_{i}^{\prime \prime}(j)\right)\right|=\sum_{i \in I}\left|T_{i}\right| \geq 0.8 \cdot \sum_{i=1}^{q} n_{i} \geq \frac{2 n d}{5 D} .
$$

Since $\left|V_{j} \cap U_{1}\right| \leq \frac{4 n_{1} \ln M}{X}$,

$$
\left|E\left(G^{\prime \prime}(j)\right)\right| /\left|V\left(G^{\prime \prime}(j)\right)\right| \geq \frac{2 n d}{5 D} \frac{X}{4 n \ln M}=\frac{d X}{10 D \ln M} .
$$

By (6) and (8), this is at most

$$
\delta_{r}(s) \frac{d X^{2}}{10 \ln M}>\delta_{r}(s) d \geq \delta_{r}(s) d_{r}(s) \geq d_{r-1}(s) .
$$

So, by the induction assumption, there is an assignment $L_{j}$ of lists of size $s$ from the set $S_{j}=$ $\left\{(j-1) R_{r-1}(s)+1,(j-1) R_{r-1}(s)+2, \ldots, j R_{r-1}(s)\right\}$ to the vertices in $V_{j} \cap U_{1}$ such that for every coloring of $V_{j} \cap U_{1}$ from these lists, the number of monochromatic edges in $G^{\prime \prime}(j)$ is at least

$$
\left|E\left(G^{\prime \prime}(j)\right)\right| \delta_{r-1}(s)=\sum_{i \in I}\left|T_{i}\right| \delta_{r-1}(s) \geq \frac{2 n d}{5 D} \delta_{r-1}(s) .
$$

Fix such assignments $L_{1}, \ldots, L_{m}$. It is important that all $S_{j}$ are disjoint. Let $F$ denote the set of all colorings of vertices in $V\left(G^{\prime \prime}\right)$ from these lists. For every $f \in F$, let $z_{i}(f)$ denote the number of monochromatic edges in $G_{i}^{\prime \prime}$ under $f$ and let $I(f)$ be the set of indices $i \in I$ such that

$$
z_{i}(f) \geq 0.5 \delta_{r-1}(s)\left|E\left(G_{i}^{\prime \prime}\right)\right|=0.5 \delta_{r-1}(s) M\left|T_{i}\right|=2 s\left|T_{i}\right| .
$$

To every vertex $v \in U_{1}-V\left(G^{\prime \prime}\right)$ we assign a list chosen at random among all subsets of size $s$ of $\left\{1,2, \ldots, M R_{r-1}(s)\right\}$ uniformly and independently from all other vertices. We will show that with positive probability this choice will satisfy the statement of our theorem.

Consider a coloring $f \in F$ and $i \in I(f)$. Say that a vertex $v \in T_{i}$ is $(i, f)$-dangerous if at least $s(r-1)$-edges from its link in $G_{i}^{\prime}$ are monochromatic in $G_{i}^{\prime \prime}$ under $f$. Let $x_{i}(f)$ denote the number of $(i, f)$-dangerous vertices in $T_{i}$. By definition, at most $s\left|T_{i}\right|$ monochromatic edges in $G_{i}^{\prime \prime}$ under $f$ belong to the links of not- $(i, f)$-dangerous vertices in $T_{i}$, and the link of each vertex in $T_{i}$ has $M$ edges. So, $x_{i}(f) \geq\left(z_{i}(f)-s\left|T_{i}\right|\right) / M$. Since $i \in I(f)$, by (15) we have

$$
x_{i}(f) \geq\left(z_{i}(f)-s\left|T_{i}\right|\right) / M \geq\left(z_{i}(f)-0.5 z_{i}(f)\right) / M=\frac{z_{i}(f)}{2 M} \geq \frac{s\left|T_{i}\right|}{M} .
$$


Since all the ground sets $S_{1}, \ldots, S_{M}$ of the lists $L_{1}, \ldots, L_{M}$ are disjoint, the probability that an $(i, f)$-dangerous vertex will get a list consisting only of $s$ colors that appear as the color of a monochromatic edge in its link in $G_{i}^{\prime \prime}$ is at least $\left(\begin{array}{c}M R_{r-1}(s) \\ s\end{array}\right)^{-1} \geq M^{-2 s}$. Hence again by the Chernoff bound, the probability of the event $C_{i}(f)$ that this occurs for fewer than $0.5 M^{-2 s} x_{i}(f)(i, f)$ dangerous vertices is at most $\exp \left\{-M^{-2 s} \frac{x_{i}(f)}{8}\right\}$. Using (16) and the facts that $i \in I$ and $s \geq 6 r \geq$ 18 , this does not exceed

$$
\exp \left\{-M^{-2 s} \frac{s\left|T_{i}\right|}{8 M}\right\} \leq \exp \left\{-0.5 s M^{-2 s-1} \frac{n_{i}}{8}\right\} \leq \exp \left\{-M^{-2 s-1} n_{i}\right\} .
$$

Since $\left|V_{j} \cap U_{i}\right| \leq \frac{4 n_{i} \ln M}{X}$, the probability of the event $C_{i}$ that at least one of $C_{i}(f)$ occurs is at most

$$
s^{4 n_{i} \ln M / X} \exp \left\{-M^{-2 s-1} n_{i}\right\}=\exp \left\{-\frac{n_{i}}{X}\left(\frac{X}{M^{2 s+1}}-4 \ln s \ln M\right)\right\} \leq \exp \left\{-\frac{n_{i}}{X}\right\} .
$$

Thus the probability of the event $C$ that at least one of $C_{i}$ occurs is at most $q \exp \left\{-\frac{n_{q}}{X}\right\}$. Recall that $q<n^{2}$ and (by Lemma 3.1(ii)) $n_{q} \geq \sqrt[r]{n d / 4}$. So by (7), $q \exp \left\{-\frac{n_{q}}{X}\right\}<1$. Thus, there exists a list assignment $L$ for $V(G)$ such that none of $C_{i}(f)$ occurs.

Let $\widetilde{f}$ be any $L$-coloring of $G$ and $f \in F$ be its restriction to $V\left(G^{\prime \prime}\right)$. We will prove that there are at least $\frac{n d}{D X}$ monochromatic edges in $\widetilde{f}$. By (14), for the number $Z$ of monochromatic edges in coloring $f$ of $G^{\prime \prime}$ we have

$$
Z \geq M \sum_{i \in I}\left|T_{i}\right| \delta_{r-1}(s) \geq M \frac{2 n d}{5 D} \delta_{r-1}(s) \geq \frac{8 s n d}{5 D} .
$$

By the definition of $I(f)$,

$$
\sum_{i \in I-I(f)} z_{i}(f)<\sum_{i \in I-I(f)} 0.5 \delta_{r-1}(s) M\left|T_{i}\right| \leq \sum_{i \in I} 0.5 \delta_{r-1}(s) M\left|T_{i}\right| \leq Z / 2 .
$$

By (16), $x_{i}(f) \geq z_{i}(f) / 2 M$ for each $i \in I(f)$. Since $C_{i}(f)$ does not occur, the number of monochromatic edges in $G_{i}$ under $\tilde{f}$ is at least $0.5 M^{-2 s} x_{i}(f)$. So by (18) and (17), the total number of monochromatic edges in $G$ is at least

$$
\sum_{i \in I(f)} 0.5 M^{-2 s} x_{i}(f) \geq \sum_{i \in I(f)} 0.5 M^{-2 s} \frac{z_{i}(f)}{2 M} \geq \frac{1}{4} M^{-2 s-1} \frac{Z}{2} \geq M^{-2 s-1} \frac{n d}{D} \geq \frac{n d}{D X} .
$$

By (6), the theorem is proved.

\section{A geometric application}

In this section, we prove Theorem 1.3. For convenience, we restate it here.

Theorem 6.1 For any finite set $X$ in the Euclidean plane and for any positive integer $s$, there is an assignment of a list of size $s$ to every point of the plane, such that whenever we color the points of the plane from their lists, there is a monochromatic isometric copy of $X$. 
Proof: Put $r=|X|$. By Theorem 1.1, it suffices to show that for any $d$ there is a $d$-regular simple $r$-uniform hypergraph whose vertex set is a finite set of points in the plane, such that the vertices of each edge form an isometric copy of $X$.

Let $\left\{v_{11}, v_{12}, \ldots, v_{1 r}\right\}$ be $r$ points in $R^{2}$ that form a copy of $X$. For each $i, 2 \leq i \leq d$, let $\left\{v_{i 1}, v_{i 2}, \ldots, v_{i r}\right\}$ be a set obtained from $X$ by applying to it a rotation. The rotations are chosen in a generic manner, to ensure that no difference between two vectors of one copy is equal to a difference between two vectors of another copy. The set of vertices of our hypergraph is the following set of $r^{d}$ sums:

$$
\left\{v_{1 j_{1}}+v_{2 j_{2}}+v_{3 j_{3}}+\cdots+v_{d j_{d}}: 1 \leq j_{t} \leq r \text { for all } t\right\}
$$

and the set of edges is the set of all $d r^{d-1} r$-tuples

$$
\left\{v_{1 j_{1}}+v_{2 j_{2}}+v_{3 j_{3}}+\cdots+v_{d j_{d}}: 1 \leq j_{t} \leq r\right\}
$$

Note that in each such edge, all summands but the $t$ th are fixed, hence every edge forms an isometric copy of $X$.

It is not difficult to check that the above hypergraph is simple, $r$-uniform and $d$-regular, and the result thus follows from Theorem 1.1.

\section{Concluding remarks and open problems}

- By (6), for any fixed $r, d_{r}(s) \leq 2^{O\left(s^{r-1}\right)}$. In other words, for any fixed $r$, the list chromatic number of any simple $n$-vertex $r$-graph with $d n$ edges is at least $\Theta\left(\ln ^{1 /(r-1)} d\right)$.

It is not difficult to check that $d_{r}(s) \geq r^{\Omega(s)}$ : for $r=2$ we know that $\chi_{\ell}\left(K_{d, d}\right)=(1+$ $o(1)) \log _{2} d$. For bigger $r$ one can use transversal designs, that is, $r$-partite $r$-uniform simple hypergraphs on $d r$ vertices, partitioned into $r$ vertex classes of size $d$ each, which have $d^{2}$ edges. One way to construct those is as follows. Let $p=d$ be a prime, and consider the $p^{2}$ polynomials of degree at most 1 over the finite field $Z_{p}$. Let each vertex class $V_{i}$ be a copy of $Z_{p}$, and assign to each linear polynomial $P$ as above an edge consisting of $P(i) \in V_{i}$ for $i=1,2, \ldots, r$. This $r$-graph is simple and $d$-regular. We claim that its list chromatic number is at most $2 \log d / \log r$. Indeed, given a list $S_{v}$ of size $s$ to each vertex $v$, assign each color in the union of all lists randomly to all vertex classes $V_{i}$ but one. This provides a proper coloring if for every vertex $v \in V_{i}$ there is a color in $S_{v}$ assigned to $V_{i}$. The probability this fails for a fixed vertex is precisely $(1 / r)^{s}$, and thus if $d^{2}(1 / r)^{s}<1$ then with positive probability there are no failures, proving the claim.

It seems plausible to conjecture that for any fixed $r, d_{r}(s)=r^{O(s)}$. This holds for $r=2$ by the results in [3] and [10] (or more generally, [4]), but remains open for any fixed $r \geq 3$.

- As mentioned in the introduction, our main result provides a linear time algorithm for computing, for a given input simple $r$-graph, a number $s$ such that its list chromatic number is 
at least $s$ and at most $f(s)$ for some explicit function $f$. The same result implies that if a simple $r$-graph is $s$-list colorable, then there is a linear time algorithm for finding a proper list coloring of it from lists of size $f(s)$. There is no such known result for ordinary coloring, even for $r=2$. Indeed, the minimum number $Q=Q(3, n)$ for which there is a polynomial time algorithm that colors properly a given 3-colorable graph on $n$ vertices by $Q$ colors, is about $\Theta\left(n^{0.207}\right)$. This is shown in [5], improving several earlier results. It is known that if $P \neq N P$ then there is no polynomial time algorithm that finds a proper 4-coloring of a 3-colorable graph (see [17], [11]), and there is no polynomial time algorithm that finds a proper $q^{(\log q) / 25_{-}}$ coloring of a $q$-colorable graph, as shown in [18]. Moreover, under some plausible hardness assumptions in Complexity Theory, there is no polynomial time algorithm that produces a proper $Q$-coloring of a $q$ colorable graph for any fixed $3 \leq q \leq Q$, as shown in [6].

- The analog of Theorem 6.1 holds for $R^{m}$ with any $m \geq 2$ (and in fact for $m \geq 3$ it is enough to only allow translations and rotations in one direction). An appropriate version works for any infinite group acting on a set.

Acknowledgment. The authors thank both referees for helpful comments and suggestions.

\section{References}

[1] N. Alon and J. H. Spencer, The Probabilistic Method, Third Edition, Wiley, 2008, xv+352 pp.

[2] N. Alon, Restricted colorings of graphs, Surveys in Combinatorics, Proc. 14th British Combinatorial Conference, Vol. 187 of London Math. Soc. Lecture Notes (K. Walker, ed.), 1993, Cambridge University Press, 1-33.

[3] N. Alon, Degrees and choice numbers, Random Structures \& Algorithms 16 (2000), 364-368.

[4] N. Alon and M. Krivelevich, The choice number of random bipartite graphs, Ann. Comb. 2 (1998), 291-297.

[5] S. Arora, E. Chlamtac, and M. Charikar, New approximation guarantee for chromatic number, Proc. 38th Annual ACM Symposium on Theory of computing, pp. 215-224, 2006.

[6] I. Dinur, E. Mossel and O. Regev, Conditional hardness for approximate coloring, Proc. of STOC 2006. Also: SIAM J. Comput. 39 (2009), 843-873.

[7] P. Erdős, R. L. Graham, P. Montgomery, B. L. Rothschild, J. Spencer, and E. G. Straus, Euclidean Ramsey theorems. I, J. Combinatorial Theory Ser. A 14 (1973), 341-363.

[8] P. Erdős, R. L. Graham, P. Montgomery, B. L. Rothschild, J. Spencer, and E. G. Straus, Euclidean Ramsey theorems. II, Infinite and finite sets (Colloq., Keszthely, 1973) Vol. I, pp. 529-557. Colloq. Math. Soc. Janos Bolyai, Vol. 10, North-Holland, Amsterdam, 1975. 
[9] P. Erdős, R. L. Graham, P. Montgomery, B. L. Rothschild, J. Spencer, and E. G. Straus, Euclidean Ramsey theorems, III, Infinite and finite sets (Colloq., Keszthely, 1973, Vol. I, pp. 559-583. Colloq. Math. Soc. Janos Bolyai, Vol. 10, North-Holland, Amsterdam, 1975.

[10] P. Erdős, A. L. Rubin and H. Taylor, Choosability in graphs, Proc. West Coast Conf. on Combinatorics, Graph Theory and Computing, Congressus Numerantium XXVI, 1979, 125157.

[11] V. Guruswami and S. Khanna, On the hardness of 4-coloring a 3-colorable graph, SIAM Journal on Discrete Mathematics, 18 (2004), 30-40.

[12] P. E. Haxell and M. Pei, On list coloring Steiner triple systems, J. Combin. Des. 17 (2009), no. $4,314-322$.

[13] P. E. Haxell and J. Verstraete, List coloring hypergraphs, Elect. J. Combinatorics 17 (2010), \#R129, 12pp.

[14] S. Janson, T. Łuczak, and A. Ruciński, Random graphs, Wiley-Interscience, New York, 2000.

[15] T. Jensen and B. Toft, Graph Coloring Problems, John Wiley and Sons Inc., New York, 1995.

[16] T. Jensen and B. Toft, Choosability versus chromaticity - the plane unit distance graph has a 2-chromatic subgraph of infinite list-chromatic number, Appendix 1 by Leonid S. Melńikov and Vadim G. Vizing and Appendix 2 by Noga Alon, Geombinatorics 5 (1995), no. 2, 45-64.

[17] S. Khanna, N. Linial and S. Safra, On the hardness of approximating the chromatic number, Combinatorica 20 (2000), 393-415.

[18] S. Khot, Improved inapproximability results for MaxClique, chromatic number and approximate graph coloring, Proc. 42nd IEEE Symp. on Foundations of Computer Science, pp. 600-609, 2001.

[19] L. Moser and W. Moser, Problem and solution P10, Canad. Math. Bull. 4 (1961), 187-189.

[20] R. Ramamurthi, Oral communication.

[21] R. Ramamurthi and D. B. West, Hypergraph extension of the Alon-Tarsi list coloring theorem, Combinatorica 25 (2005), 355-366.

[22] V. G. Vizing, Coloring the vertices of a graph in prescribed colors Diskret. Analiz. No. 29, Metody Diskret. Anal. v. Teorii Kodov i Shem 101 (1976), 3-10 (Russian). 